\title{
The multiple internal clocks of marine animals
}

Internal circadian clocks allow organisms to anticipate the regular environmental changes associated with the cycles of day and night. These circadian clocks are driven by a set of regulatory genes that comprise negative and positive transcriptional and translational feedback loops, oscillating in activity over a period of $24 \mathrm{~h}$. Many of these genes are shared between insect and mammalian models, suggesting a common origin of circadian clocks among animals.

Many organisms also exhibit rhythms of longer or shorter period lengths. For example, species inhabiting coastal environments, from brown algae and corals to worms and vertebrates, synchronize their maturation and spawning not only to particular times of day, but also to a particular moon phase or specific seasons within a year, responding to moonlight and photoperiod cues, respectively. Two recent studies shed light on the relationship of the circadian clock to other endogenous clocks.

A study led by Kristin Tessmar-Raible (University of Vienna, Austria) examined the interactions between the endogenous

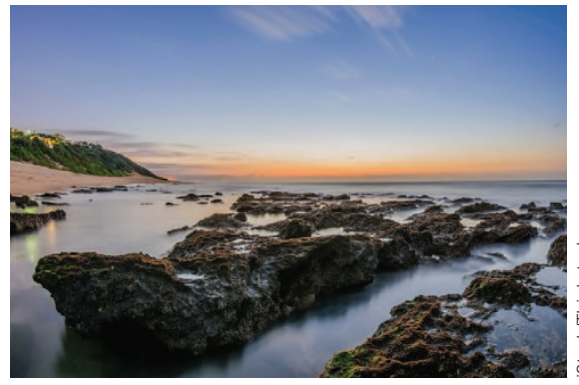

circadian and circalunar (monthly) clocks in the bristle worm (Platynereis dumerilii). In a distinct nucleus of the worm's forebrain, levels of core circadian genes oscillate not only over $24 \mathrm{~h}$, but also across different phases of the lunar month. The period length and strength of locomotor behaviors that are controlled by the circadian clock, such as spawning, change over different phases of the circalunar clock as well (Cell Rep. doi:10.1016/ j.celrep.2013.08.031; published online 26 September 2013). "Our results suggest that the bristle worm possesses independent, endogenous monthly and daily body clocks that interact," says Tessmar-Raible.
Another study shows that the intertidal crustacean Eurydice pulchra, also known as the sea louse, is guided by not only circadian rhythms but also tidal rhythms (Curr. Biol. doi:10.1016/j.cub.2013.08.038; published online 26 September 2013). Metabolism, reproduction and swimming behavior show cycles of $12.4 \mathrm{~h}$ in parallel to their 24-h cycles. Charalambos Kyriacou (University of Leicester, UK) and his colleagues at MRC Laboratory of Molecular Biology (Cambridge, UK) and Aberystwyth University (UK) reported that the tidal rhythm meets the three criteria that define a biological clock: it is sustained in constant conditions, can be entrained by the appropriate stimuli and shows temperature compensation. They further showed that environmental and molecular signals that interfere with circadian timing do not affect tidal timing. Says Kyriacou, "The identification of the tidal clock as a largely separate mechanism now presents us with an exciting new perspective on how coastal organisms define biological time."

Kara Rosania

\section{BETTER ANESTHESTA FOR ZEBRAFISH}

Millions of zebrafish (Danio rerio) are used in laboratories around the world, often as part of developmental studies. Zebrafish embryos are transparent and develop externally, facilitating observation and manipulation of the developmental process and leading to greater understanding of developmental defects and diseases. As research subjects, fish are often anesthetized for handling, sample collection and surgical procedures (e.g., fin clipping) and as the first step in euthanasia. Various anesthetic agents may be used for this purpose, but despite routine use, no studies have formally assessed how well the agents are tolerated by fish or whether they trigger aversive behavioral responses. The lack of information impedes the establishment of best practices for zebrafish anesthesia. Joanna C. Murrell, a lecturer in veterinary anesthesia at University of Bristol (Langford, UK), explained, "With tens of millions of fish used in science around the world, it is very important that the anaesthetics used... are the most humane available and do not themselves cause a stress response."

Murrell worked with Gareth Readman (Brixham Environmental Laboratory, AstraZeneca, Devon, UK) to evaluate nine of the most commonly used fish anesthetics. They carried out preference testing, exposing adult zebrafish of both sexes to water containing each agent at $50 \%$ of its standard recommended dose in a flow-through chemotaxic choice chamber and using video tracking software to quantify aversive swimming behavior related to each anesthetic compared with untreated water. They found that seven of the nine anesthetics were aversive, including MS222 (ethyl 3-aminobenzoate methanesulphate) and benzocaine, two of the most often used (PLOS ONE 8, e73773; 2013). The authors recommend that for ethical best practice, aversive compounds should not be used routinely for anesthesia or for the first step of euthanasia of adult zebrafish.

Two other agents, etomidate and 2,2,2 tribromoethanol (TBE), did not induce aversive behavioral responses. In previous investigations, etomidate has not caused negative allergenic, tissue irritation or blood chemistry responses in fish, although metomidate hydrochloride, an etomidate derivative, has been reported to have negative effects in human and veterinary medicine. Although TBE did not elicit an aversive reaction, the authors caution that improper storage can lead to the formation of potentially harmful degradation products. The authors conclude that etomidate is best suited for routine anesthesia of zebrafish.

The results could improve the welfare standards for zebrafish used in research. In contrast to the many advances in anesthesia for laboratory mammals, this is one of the first studies to assess the welfare implications of anesthesia in fish. 